\title{
Acute Care SINS: Surgical Insights for the
}

\section{Non-surgeon}

\section{Chapter 5: Small Bowel SINS}

\author{
Rachel G. Khadaroo MD PhD, Kamran Fathimani MD, Peter G. Brindley MD
}

\begin{abstract}
About the Authors
Rachel Khadaroo and Peter Brindley are members of the Division of Critical Care Medicine, and Rachel Khadaroo and Kamran Fathimani (far right) are members of the Division of General Surgery, all at the University of Alberta, in Edmonton, Alberta. Correspondence may be directed to peter.brindley@albertahealthservices.ca.
\end{abstract}

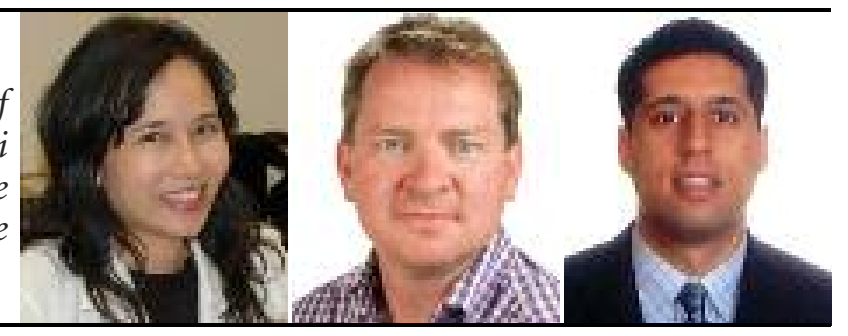

\section{Summary}

"Surgical Insights for the Non-surgeon," or SINS, is composed of several short chapters intended to cover fundamental surgical knowledge for non-surgeons. The authors focus on surgical pearls, operative insights, and applied anatomy. In Chapter 5 of this series, the authors discuss the small bowel - its anatomy, its obstruction, and small bowel/mesenteric ischemia.

\section{Résumé}

L'ouvrage « Surgical Insights for the Non-surgeon » ou SINS (aperçu de chirurgie pour le nonchirurgien) se compose de plusieurs courts chapitres conçus pour couvrir les connaissances fondamentales en matière de chirurgie chez ceux qui ne sont pas chirurgiens. Les auteurs se concentrent sur des «trésors de sagesse » tirés de leur expérience personnelle en chirurgie, certaines idées en matière d'interventions, et sur l'anatomie appliquée. Dans le chapitre 5, les auteurs s'intéressent à l'intestin grêle - notamment à son anatomie, aux occlusions et à l'ischémie du grêle, dont l'ischémie mésentérique.

"Measure thrice, think twice, cut once."

- Old adage from carpentry ... and just as relevant to surgery

\section{Anatomy}

The small bowel is divided into duodenum (approximately $30 \mathrm{~cm}$ ), jejunum (approximately $300 \mathrm{~cm}$ ), and ileum (approximately $100 \mathrm{~cm}$ ). The duodenum is divided into four parts: the first part or bulb; the second part or descending portion; the third part or transverse portion; and the fourth part or portion ascending to the ligament of Treitz. Duodenal arterial supply is primarily via the gastroduodenal artery. It courses posterior to the duodenal bulb. Therefore, an ulcer that penetrates the posterior wall of the first part of the duodenum can cause massive hemorrhage. This may necessitate surgery (see Chapter 4: Stomach SINS ${ }^{1}$ ). When there is $<200 \mathrm{~cm}$ of small bowel, there is risk of short bowel syndrome (not enough bowel to absorb the nutritional requirements). Therefore, care is taken to save an adequate length following a large or multiple bowel resections.

\section{Small Bowel Obstruction Etiology}

By anatomy:

- Obstruction from extraluminal causes: adhesions, hernias, carcinomas, and abscesses

- Obstruction intrinsic to the bowel wall: primary tumours, hematoma, inflammation, Crohn's disease

- Intraluminal obstruction: gallstones, enteroliths, foreign bodies, and bezoars

\section{By incidence:}

- Adhesions $(\approx 50-75 \%)$

- Hernias ( $\approx 10-25 \%)$

- Crohn's disease (increasing frequency, $>5 \%$ )

- $\quad$ Neoplasm $(<5 \%)$ 


\section{Pathophysiology}

- Early stages: increased intestinal motility; may present as diarrhea

- Later stages

- Small intestine dilates, and motility decreases

- Failure to pass flatus and feces

- Extravascular fluid gain and intravascular fluid loss

- Even later stages

- Vomiting (metabolic alkalosis, hypochloremia, and hypokalemia)

- Hypotensive shock (dehydration, oliguria, \pm hypotension)

- Sepsis (due to translocation of gut bacteria)

\section{Diagnosis}

\section{History and physical:}

- Nausea and vomiting, abdominal distension

- Cramping abdominal pain

- Volume depletion: tachycardia, postural blood pressure changes, shock

\section{Radiological and laboratory examinations:}

- Abdominal radiography

- $\quad$ Still the best initial radiological test (Figures 1 and 2)

- Look for air-fluid levels and small bowel dilation

- Abdominal/pelvic computed tomography (CT)

- May help differentiate partial from complete obstruction

- Assists with pinpointing location and cause

- Useful to identify bowel strangulation

- Barium swallow

- Can show the level and possible causes of small bowel obstruction (SBO)

- Not commonly used nowadays

\section{- Enteroclysis}

- Oral insertion of tube into duodenum

- Instill air and barium directly into the small intestine

- Magnetic resonance imaging (MRI): no better than CT

- Laboratory tests: for assessing volume depletion or ischemia (acidosis, lactate)

\section{Strangulating Obstruction}

- Usually implies a closed-loop obstruction; vascular supply compromised - ischemia results

- Immediate surgery to prevent bowel necrosis

\section{Treatment of SBO}

\section{Medical:}

- Intravenous (IV) fluid replacement (“drip”): if making

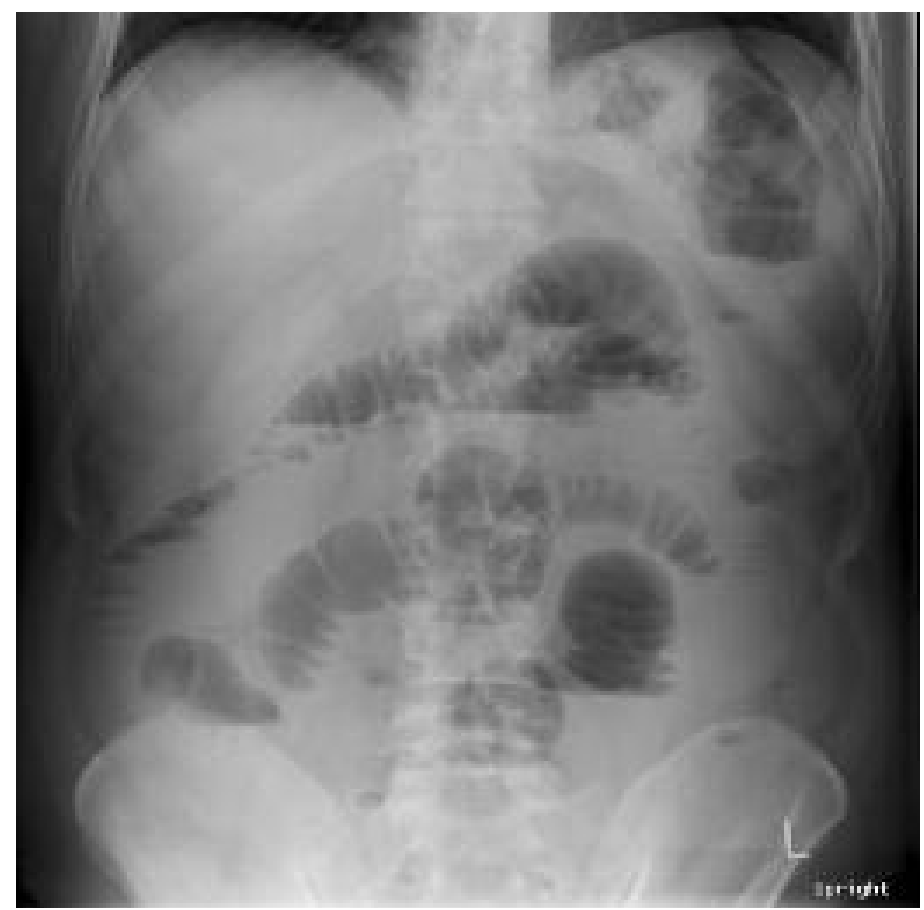

Figure 1. Upright abdominal radiograph showing multiple air-fluid levels suggestive of small bowel obstruction.

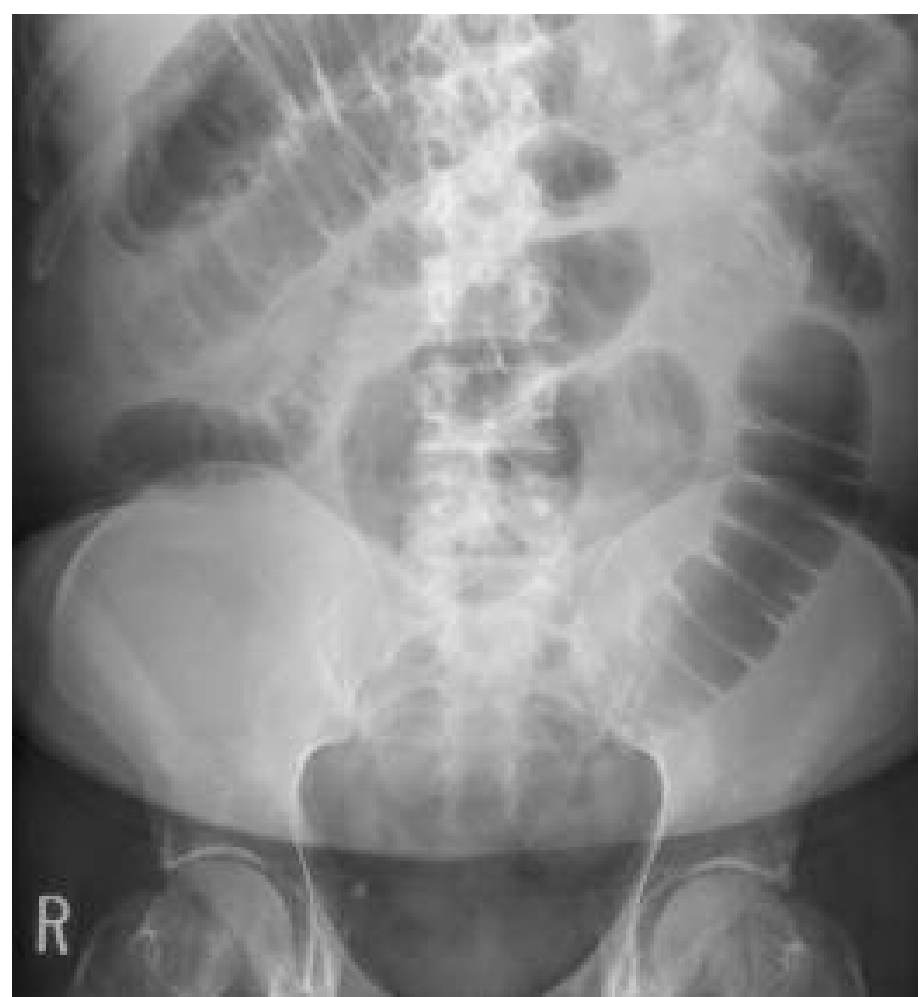

Figure 2. Supine abdominal radiograph showing dilated small bowel loops suggestive of small bowel obstruction.

urine, you should add IV potassium

- Nasogastric tube ("suck")

- Reduces aspiration

- Reduces further distension from swallowed air 


\section{Surgical Pearls: Summary of SBO}

Pearl 1: Classification of SBO Etiology

\section{Common:}

- Adhesions

- Hernias

\section{Uncommon:}

- Crohn's disease

- Small bowel neoplasm

- Meckel's diverticulum

- Intussusception

- Gallstone ileus

- Congenital adhesions

- Appendicitis

- Cecal cancer

- Small bowel volvulus

- Foreign body

\section{Pearl 2: Be Alert to Common Causes of SBO}

- Adhesions: during history taking, ask about all previous abdominal surgeries

- Hernias: during physical examination, inspect for abdominal scars and palpate for hernias (both abdomen and groin)

\section{Pearl 3: Management of SBOS - Adhesions and Hernias}

- Adhesions

- $\quad$ NPO (nothing by mouth), nasogastric tube, IV fluids, serial abdominal examination and radiography

Surgery if peritonitis or failure of medical management

- Hernias: surgical repair of abdominal wall or inguinal hernia

\section{Pearl 4: Management of SBOs - Uncommon Etiologies}

- SBO despite no previous abdominal surgeries, or abdominal wall hernias, usually means a pathological cause and, therefore, the need for surgery

- Broad-spectrum antibiotics not routinely recommended for uncomplicated SBO

- 60-85\% recover from partial SBO with just "suck and drip" therapy

- Serial abdominal radiography

\section{Surgical:}

- Notify surgery if any of the following occur:

- Ischemia, shock, increasing white blood cell (WBC) count

- $\quad$ Complete SBO >24-72 hours

- Peritonitis

- Worsening results on serial abdominal radiographs

- Increasing abdominal pain (with concerns of bowel ischemia)

- $\quad$ Failure to resolve within 24 hours of medical management: "Never let the sun rise and set on a complete bowel obstruction"

- In short, keep in contact with your friendly surgeon

\section{Small Bowel Ischemia/Mesenteric Ischemia}

- Rare but life threatening

- All physicians need to know the basics

- Can occur gradually; better tolerated if collateral blood supply forms over time

- Can occur acutely; associated with high mortality

\section{Three Main Causes}

\section{Embolus:}

- Common cause of mesenteric ischemia

- Usually clot from cardiac source embolized to superior mesenteric artery (SMA)

- Occlusion usually distal to the SMA origin because the clot lodges in the smaller branches

\section{Thrombosis:}

- Less common

- Usually from plaque occlusion at the vessel's origin

- Often less severe due to chronicity (i.e., collaterals exist)

Acute non-occlusive mesenteric insufficiency (NOMI):

- Associated with hemodynamic compromise (e.g., sepsis, heart failure)

- Global decrease in blood supply to the bowel

\section{Clinical Presentation}

- Pain out of proportion to physical examination!

- However, initial presentation may be only mild tenderness, with or without peritonitis

- Tenderness increases over time (therefore, serial examinations are very useful)

- May have blood in stool from sloughing mucosa

\section{Laboratory Investigations}

- Elevated WBC count

- Metabolic acidosis and lactate (serial electrolytes or arterial blood gases)

\section{Radiology}

- AXR and CT may show fluid-filled bowel-loops and bowel edema

- Arteriogram or contrast CT may show the arterial cut-off (Remember IV contrast put kidneys at risk)

- Gas in bowel wall (pneumatosis intestinalis) is a late finding: indicates bacterial spread into tissue 
- Gas in portal system (portal venous gas) is a very bad sign: indicates bacterial spread into the blood supply

\section{Differential Diagnosis}

- Acute pancreatitis

- Perforated viscus

- Ruptured aneurysm

- Kidney stone

\section{General Treatment}

- Requires laparotomy for diagnosis ("peak and shriek") and treatment ("damage control")

- Extensive necrosis has poor prognosis

- Necrotic bowel is resected

- Typical to perform a "second-look laparotomy" in 24-48 hours to reassess the bowel

\section{Specific for Occlusive Mesenteric Disease (Embolus or Thrombosis)}

- Primarily surgical treatment with embolectomy or thrombectomy \pm arterial reconstruction

- Alternative therapeutic options during angiography

- Intra-arterial vasodilators or thrombolytic agents

- Angioplasty

- Placement of a vascular stent

- Embolectomy

- Early discussion with a vascular surgeon on best approach

\section{Specific for Non-occlusive Mesenteric Insufficiency}

- Usually in patients that have had hemodynamic instability (e.g., patients in intensive care unit or cardiac patients)

- Difficult diagnosis if patient is sedated and intubated

- Signs and symptoms similar to occlusive mesenteric disease

\section{Treatment:}

- $\mathrm{ABCs}$ (airway, breathing, and circulation) and treat underlying cause

- $\quad$ Fluid resuscitate and limit vasoconstrictors

- Antibiotics to combat bacterial translocation

- Surgery for dead gut

- Generally poor prognosis

\section{Mesenteric Venous Occlusion}

- Associated with medical illnesses

- Portal hypertension, post-operative state

- Intra-abdominal inflammation (pancreatitis, inflammatory bowel disease)

- Hypercoagulable states (neoplasm, protein C and S deficiencies)
- Less acute presentation

- Contrast CT for diagnosis

- Shows thickened bowel wall

- Slow passage of IV contrast into the portal system

- Lack of opacification of the portal vein

- Arteriography may show venous congestion and poor filling of portal system

\section{Treatment:}

- Hemodynamic support

- Systemic anticoagulation

- Surgery for peritonitis and suspected necrotic bowel: venous thrombectomy is rarely performed (due to poor success rate)

- Good prognosis due to (1) collateral venous drainage and (2) mesenteric veins recanalization

\section{Chronic Mesenteric Insufficiency}

- Also known as intestinal angina

- Almost always in elderly patients with diffuse atherosclerosis

- Otherwise, suspect celiac artery compression by diaphragm's median arcuate ligament

- Causes post-prandial abdominal pain (mesenteric supplydemand imbalance)

\section{Diagnosis:}

- Doppler sonography of proximal superior mesenteric artery and celiac artery

- Arteriography for definitive diagnosis: requires occlusion of at least two of the three major mesenteric arteries

- MRI useful in younger patient to show compression of the proximal celiac artery

\section{Treatment:}

- Angiographic: balloon angioplasty or stent placement

- Surgery: transaortic endarterectomy or bypass graft

- From supraceliac artery to the celiac artery and superior mesenteric

- Or retrograde bypass from infrarenal aorta or iliac artery

\section{Works Cited or Consulted}

1. Khadaroo RG, Fathimani K, Brindley PG. Acute care SINS: surgical insights for the non-surgeon. Chapter 4: stomach SINS. Can J Gen Intern Med 2013;8(2):59-64.

2. Townsend CM, Beauchamp RD, Evers BM, Mattox KL. Sabiston Textbook of Surgery, 19th edition. Philadelphia (PA): Elsevier Saunders; 2012. 